\title{
Universidades nos sistemas de inovação: produção de pesquisa científica nas universidades federais do nordeste do Brasil ${ }^{\dagger}$
}

Tulio Chiarini*

Karina Pereira Vieira**

\begin{abstract}
Resumo: $\mathrm{O}$ crescimento econômico da região nordeste e a atração de empresas para a mesma, na última década, são parte importante de uma evolução única na história da região. Paralelamente a isso, a região nordeste é a segunda região do país em número de universidades federais. Neste artigo, parte-se do conceito de universidade como agente fundamental da constituição de um sistema de inovação forte, capaz de dar suporte ao desenvolvimento. Assim, é realizado um mapeamento das universidades federais do nordeste, observando sua produção científica, os grupos de pesquisa das mesmas, e também sua interação com o setor produtivo. Nota-se que UFPE, UFBA e UFC são os principais agentes na produção de conhecimento da região, embora apresentassem produção científica relativamente baixa, se comparada a outras regiões do país. Tal mapeamento é importante no sentido de avançar na discussão sobre a importância da inclusão das universidades federais em um programa maior de desenvolvimento científico e tecnológico brasileiro.
\end{abstract}

Palavras-chave: Universidades; Produção de conhecimento; Sistema de inovação.

Classificação JEL: O33; I23; I28.

\footnotetext{
$\bar{\dagger}$ Os autores gostariam de agradecer às contribuições do Prof. Dr. Luiz Eugênio V. Pasin do Instituto de Engenharia de Produção e Gestão da Universidade Federal de Itajubá (IEPG/UNIFEI), o qual fez uma avaliação criteriosa deste. No entanto, o eximimos de responsabilidades pelas imperfeições que eventualmente ainda possam persistir.

* Mestre em Economia pela Universidade Federal do Rio Grande do Sul (UFRGS) e mestre em Managment, Innovazione e Ingegneria dei Servizi pela Scuola Superiore Sant'Anna di Studi Universitari e di Perfezionamento (MAINS/SSSP). Professor do Instituto de Engenharia de Produção e Gestão da Universidade Federal de Itajubá (IEPG/UNIFEI) e doutorando em Economia pelo Instituto de Economia da Universidade Estadual de Campinas (IE/Unicamp). Endereço eletrônico: tuliochiarini@unifei.edu.br.

${ }^{* *}$ Mestre em Economia pelo Centro de Desenvolvimento e Planejamento Regional da Universidade Federal de Minas Gerais (Cedeplar/UFMG). Analista socioeconômica do Instituto Brasileiro de Geografia e Estatística (IBGE). Endereço eletrônico: karina.pvieira@gmail.com.
} 


\section{Introdução}

De 1998 a 2008, a região nordeste do Brasil apresentou crescimento considerável, com um aumento de $45 \%$ do PIB a preços constantes ${ }^{1}$. Além disso, estima-se que, entre 2000 e 2008, o bom desempenho econômico da região deveu-se a expansão de $44,5 \%$ do número de empresas industriais e ao aumento de $55,5 \%$ do número de pessoas ocupadas na indústria nordestina ${ }^{2}$. Trata-se, portanto, de um período único na história da região, reconhecida pelo potencial turístico e agroindustrial, e que, no entanto, atraiu, nesse período, empresas industriais de grande porte, como Ford, Nestlé, Kraft, Unilever, Perdigão e outras. Tal evolução também fica evidente quando se observa o investimento em inovação das empresas localizadas na região. Conforme informações da Pesquisa de Inovação Tecnológica (PINTEC) do Instituto Brasileira de Geografia e Estatística (IBGE), o investimento em atividades inovativas passou de $\mathrm{R} \$ 990,15$ milhões, em 2000, para $\mathrm{R} \$ 2,08$ bilhões, em 2008, demonstrando o esforço das empresas nordestinas em se empenharem em processos inovativos.

Tais investimentos industriais atraídos para a região nordeste têm efeitos que vão além da geração de emprego e renda e do estímulo ao consumo no curto prazo (Vieira; Albuquerque, 2007). No longo prazo, podem se desenvolver conhecimentos tácitos na produção de determinados setores, uma vez que a mão de obra local tende a se especializar, o que também amplia a sua capacidade de absorver conhecimentos técnicos, permitindo maior capacidade de difundir tecnologia. Dessa forma, o estreitamento das relações universidade-estado-empresa apoiados por outras instituições, como centros de pesquisa (públicos e/ou privados), demais estabelecimentos de ensino (como ensino técnico) e sistema de financiamento e crédito, são cruciais. No entanto, mesmo reconhecendo a necessidade do estreitamento das relações entre as universidades e as empresas, sabe-se que em sistemas de inovação intermediários ou imaturos, tais relações são débeis (Suzigan; Albuquerque, 2008). Isso ocorre pois, mesmo em instituições de pesquisa e ensino consolidadas, há constrangimentos na mobilização de recursos humanos necessários para gerar expressivos circuitos de retroalimentação positiva entre as dimensões científicas e tecnológicas; além disso, as firmas têm restrito envolvimento em atividades inovativas (Rapini, et al., 2009).

1 Dados disponíveis no Ipeadata. http:// www.ipeadata.gov.br/.

2 Informações calculadas a partir de dados da Pesquisa Industrial Anual (PIA), do IBGE.

Revista Economia \& Tecnologia (RET)

Vol. 8(1), p. 137-160, Jan/Mar 2012 
Desse modo, é fundamental que as universidades e os institutos de pesquisa presentes na região sejam capazes dar suporte ao crescimento observado, a fim de que se formem bases sólidas e sustentáveis para o desenvolvimento regional. A excelência em capacitação tecnológica e científica das universidades, associada às demais instituições, pode constituir polos tecnológicos como é o caso do Porto Digital de Recife, referência nacional em tecnologia de informação (Vieira; Albuquerque, 2007).

Considerando o exposto acima, faz-se interessante caracterizar as instituições de ensino superior (IES) federais do nordeste, bem como seu relacionamento com o setor produtivo. Este trabalho encontra-se dividido em três seções, além desta introdução e das considerações finais. A primeira seção apresenta uma revisão da literatura sobre a importância das universidades para o desenvolvimento. Na seção seguinte é feita a contextualização das IES brasileiras, a fim de delimitar a análise às IES do nordeste. Em seguida, na Seção 4, partese da investigação acerca da desigualdade das IES federais nordestinas para então fazer um retrato da geração de conhecimento científico e tecnológico das IES federais do nordeste, apresentando a proporção de pesquisadores, grupos de pesquisa, produção bibliográfica, produção técnica de cada IES federal do nordeste, a partir dos dados disponibilizados pelo Diretório de Grupos de Pesquisa do CNPq, cuja importância reside no fato de apresentar informações sobre os recursos humanos constituintes de cada grupo (pesquisadores, estudantes e técnicos), as linhas de pesquisa em andamento, os setores envolvidos, a produção científica e tecnológica, por exemplo. Dessa forma, é possível acompanhar e avaliar a capacidade instalada de pesquisas nas universidades da referida região. Nessa mesma seção busca-se qualificar as relações entre os Grupos de Pesquisa das IES federais do nordeste e as empresas. Será verificado no decorrer do artigo que as IES federais não formam instituições homogêneas, sendo que a Universidade Federal de Pernambuco (UFPE), a Universidade Federal da Bahia (UFBA) e a Universidade Federal do Ceará (UFC) são as que se destacam em termos de recursos voltados à produção de conhecimento. Por último, são feitas considerações acerca dos resultados obtidos e de suas implicações para o desenvolvimento da região. 


\section{Papel das universidades no desenvolvimento}

No caso das universidades, além do processo de criação e disseminação de novos conhecimentos científicos e novas tecnologias, através de pesquisa básica, pesquisa aplicada, desenvolvimento e engenharia, cabe-lhes também a formação de mão de obra qualificada para suprir a demanda do setor produtivo. Com o ensino superior, o quadro de pesquisadores se renova e o conhecimento se atualiza (Marcovitch, 1999).

As instituições de ensino superior (IES) podem ser encaradas, portanto, como agentes estratégicos para o catch-up pois contribuem ao desenvolvimento científico-tecnológico não só da região em que estão inseridas, mas também do país, o qual é um dos principais motores que garante a possibilidade de inovação e leva a mudanças econômicas e sociais. O desenvolvimento científicotecnológico é um processo dinâmico e é resultado da coletividade, da interação entre diferentes agentes econômicos, especialmente, pois os novos paradigmas tecnológicos ${ }^{4}$ estão permeados por conhecimentos de natureza científica que se encontram muito próximos da fronteira do conhecimento, daí a importância em fomentar o papel ativo das IES e o estreitamento das relações universidade-empresa-estado de forma bem sucedida, como o caso recifense.

A geração de novos conhecimentos tem seu principal suporte no sistema público de pesquisa, fruto da pós-graduação (Marcovitch, 1999), e tem encontrado respaldo na demonstração de interesse tanto de empresas privadas quanto do Estado. Essa simbiose entre esses três agentes foi apontada por Sábato e Botana (1968) e ganhou novo destaque com a 'hélice tríplice' (Leydesdorff; Etzkowitz, 1998; Leydesdorff, 2000; Etzkowitz et al., 2000; Etzkowitz, 2003; Leydesdorff; Meyer, 2006), a qual reconhece que tais atores podem assumir o papel um dos outros, a fim de que o sistema inovativo funcione adequadamente, complementando o arcabouço heurístico dos sistemas de inovação (SI). Dessa forma, a relação empresa-universidade - como a colaboração para pesquisa e desenvolvimento (P\&D), por exemplo - pode ser justificada uma vez que reduz as incertezas intrínsecas ao processo de inovação (Tether, 2002), diluindo os riscos relacionados à incerteza das atividades inovativas (Hagedoorn et al., 2000). A 3 Termo que se refere ao esforço de adesão das inovações tecnológicas ao paradigma vigente, diminuindo a distância até a fronteira tecnológica.

4 A concepção de paradigma tecnológico foi proposta por Giovanni Dosi, no ínicio dos anos 1980, ao afirmar que esse é um pacote de procedimentos que visam à orientação da investigação e da pesquisa sobre um problema tecnológico, definindo, dessa forma, o contexto, os objetivos a serem alcançados e os recursos a serem utilizados (Kupfer, 1996). 
relação pode ser benéfica para as empresas, ao gerar o desenvolvimento de capacidades, aprendizagem e aquisição de conhecimento e tecnologias (Marques et al., 2007),contribuindo para que projetos industriais sejam concluídos e/ou auxiliando a implementação de outros novos (Cohen et al., 2002). Outros produtos de importância econômica, sejam eles mais ou menos (in)tangíveis, apresentam diversas configurações e modificam-se ao longo do tempo e conforme a indústria/setor de análise (Chiarini; Vieira, no prelo). Informação científica e tecnológica, equipamentos e instrumentos, habilidades/capital humano, network de capacidades científicas e tecnológicas e protótipos de novos processos e produtos são alguns exemplos dessa gama de produtos que podem ser gerados com a pesquisa das IES, intensificada com a relação universidade-empresa (Mowery; Sampat, 2005).

A literatura nacional apresenta estudos que buscam analisar a relação universidade-empresa, como em Plonski (1995), Gomes (2001), Dagnino (2003), Dagnino e Gomes (2003), Baldini e Borgonhoni (2007), Marques et al. (2007), Rapini e Righi (2007), Zawislak e Dalmarco (2009), somente para mencionar alguns. Existem também aqueles cujo enfoque se da sobre a 'hélice tríplice', como Dagnino (2003), Almeida (2004; 2005), Mello (2004), Abdalla et al. (2009) e Calvosa et al. (2009). Outros trabalhos visam verificar o papel das IES brasileiras nos sistemas de inovação, como Albuquerque (1996), Albuquerque (1998), Cruz (2004), Albuquerque et al. (2005b), Theis et al. (2006), Rapini (2007), Renault et al. (2008), Póvoa e Rapini (2009), Rapini et al. (2009), Esteves e Meirelles (2009), Mello et al. (2009) e Suzigan e Albuquerque (2009). O mesmo tem sido feito em âmbito regional por Vasconcelos e Ferreira (2000), Silva et al. (2000), Rapini e Campos (2004), Albuquerque et al. (2005a), Rapini et al. (2006), Righi e Rapini (2006), Chiarini et al. (2010), Chiarini et al. (no prelo), ao terem como foco de análise o estado de Minas Gerais. Não foram encontrados estudos similares tendo como objeto de análise os estados que compõem o nordeste brasileiro, portanto, busca-se preencher essa lacuna com a análise que se apresenta a seguir.

Esse artigo complementa os projetos de pesquisa de Chiarini et al. (2010) e Chiarini e Vieira (no prelo), cujo âmbito maior é entender o papel das IES públicas brasileiras em esculpir o sistema nacional de inovação. Dessa forma, o objetivo geral desse artigo é encontrar elementos que possam contribuir 
à pesquisa realizada por esses autores, demonstrando que existe uma limitação estrutural na produção de conhecimento nas IES federais do nordeste que acaba por caracterizar o sistema nordestino de inovação, ao que tudo indica, em estágio embrionário, onde a produção de novos conhecimentos tecnocientíficos e as relações universidade-empresa são menores, vis-à-vis outras regiões do país.

\section{As universidades federais como foco de análise}

Existem, no Brasil, universidades com maior foco na geração e produção de conhecimento e invenções que outras. A grande maioria das instituições privadas nacionais ${ }^{5}$ de ensino superior está focada apenas em ensino. Considerando-se que não é a intenção deste trabalho discutir a relevância ou a qualidade da formação oferecida pelas IES privadas brasileiras, cabe aqui apenas destacar que instituições privadas de ensino superior que se dedicam à pesquisa científica são raras exceções, ficando a produção de conhecimento científico a cargo principalmente das universidades públicas ${ }^{6}$.

Um fator que aponta para a diferença de funções entre as universidades privadas e públicas é o diferencial em termos de recursos humanos. A partir da Sinopse Estatística da Educação Superior de 2008 do Instituto Nacional de Estudos e Pesquisas Educacionais Anísio Teixeira, vê-se que do total de docentes universitários brasileiros, $69 \%$ apresentam o título de mestrado ou doutorado. Desagregando esse valor entre as universidades, tem-se que $75 \%$ dos docentes nas universidades públicas apresentam essas titulações. Nas universidades federais, a participação de docentes com o título de mestre ou doutor é ainda maior, 79\%. Finalmente, nas universidades privadas, o número de mestres ou doutores docentes representa $61 \%$ da titulação nessas instituições. $O$ fato de haver maior representatividade de mestres e doutores nas universidades públicas, quando comparadas às universidades privadas, reafirma a maior capacidade de produção de conhecimento nas IES públicas, já que aqueles são profissionais altamente qualificados, convivendo e trabalhando em um ambiente propício à produção de conhecimento (Chiarini et al., 2010).

Analisando somente o número de doutores, os quais não são apenas aqueles que receberam o mais elevado nível de qualificação educacional formal 5 Universidades privadas correspondem ao somatório das universidades particulares e comunitária/confessional/ filantrópica. 6 Universidades públicas correspondem ao somatório das universidades federais, estaduais e municipais.

Revista Economia \& Tecnologia (RET)

Vol. 8(1), p. 137-160, Jan/Mar 2012 
possível, mas compõem também a parcela dos recursos humanos treinada especificamente para realizar pesquisa e desenvolvimento, tendo, portanto, papel estratégico no processo de produção e transmissão de conhecimentos (codificados e tácitos) e tecnologias (Viotti, 2010), tem-se que dos docentes de todas as IES brasileiras ${ }^{7}$, apenas $23 \%$ apresentavam o título de doutor, enquanto que nas IES públicas esse valor sobe para $44 \%$; nas privadas cai para $13 \%$.

Quando se observa a distribuição das universidades federais pelo território brasileiro, nota-se que a região nordeste é bastante privilegiada, contando com $25 \%$ das IES federais de todo país (no entanto, representa em média 1,55 universidades por estado), ficando atrás somente da região sudeste, a qual concentra 34\% das IES federais (com 4,75 universidades em média por estado) (Tabela 1). No entanto, essa concentração de universidades federais no nordeste não reflete em produção de novos conhecimentos capaz de alavancar o desenvolvimento da região, como será visto adiante.

Tabela 1 - Valor absoluto e porcentagem de universidades federais por região, e média de universidades federais por estado, 2008

\begin{tabular}{|c|c|c|c|}
\hline & Total & $\%$ & $\begin{array}{l}\text { Média de universidade } \\
\text { federal por estado }(*)\end{array}$ \\
\hline Brasil & 55 & 100 & 2,03 \\
\hline Sudeste & 19 & 35 & 4,75 \\
\hline Nordeste & 14 & 25 & 1,55 \\
\hline Sul & 9 & 16 & 3,00 \\
\hline Norte & 8 & 15 & 1,14 \\
\hline Centro-oeste & 5 & 9 & 1,25 \\
\hline
\end{tabular}

Fonte: Elaboração própria a partir dos dados da Sinopse Estatística da Educação Superior 2008, INEP.

Nota: (*) São 26 estados da federação mais um Distrito Federal; para fins de análise considerou-se 27 estados.

Para fins de demarcar o objetivo de análise, encontram-se no nordeste as seguintes universidades federais: Universidade Federal de Alagoas (UFAL), Universidade Federal da Bahia (UFBA), Universidade Federal do Ceará (UFC), Universidade Federal de Campina Grande (UFCG), Universidade Federal Rural do Semi-Árido (UFERSA), Universidade Federal do Maranhão (UFMA), Universidade Federal da Paraíba (UFPB), Universidade Federal de Pernambuco (UFPE), Universidade Federal do Piauí (UFPI), Universidade Federal do Rio Grande do Norte (UFRN), Universidade Federal Rural de Pernambuco (UFRPE), Universidade Federal de Sergipe (UFS) e Universidade Federal do Vale do

7 Consideram-se aqui todas as IES públicas e privadas do Brasil de acordo com a Sinopse Estatística da Educação Superior de 2008 do Instituto Nacional de Estudos e Pesquisas Educacionais Anísio Teixeira. 
São Francisco (UNIVASF).

\section{Geração de conhecimento nas IES federais do nordeste}

A fim de caracterizar as IES da região nordeste, bem como suas interações com o setor produtivo, esta seção parte da mensuração do grau de desigualdade entre as mesmas. Tal medida é importante, pois se trata de um ponto de partida para traçar o perfil das IES nordestinas, que são atores importantes para a constituição de um sistema de inovação capaz de dar suporte ao desenvolvimento da região.

\subsection{Coeficiente de desigualdade entre as IES federais do nordeste}

A partir de um índice capaz de mensurar desigualdades entre uma distribuição, pode-se quantificar o quão desiguais são as universidades federais do nordeste no quesito pesquisadores, Grupos de Pesquisa, produção bibliográfica e produção técnica. Embora o objetivo principal de Albuquerque et al. (2001) fosse outro, eles realizaram vários exercícios estatísticos, como o cálculo do coeficiente de desigualdade de Gini para a produção científica tecnológica para os municípios brasileiros em 2000, chegando-se ao valor de 0,9873 para a produção tecnológica (proxy patentes), 0,9958 para a produção científica (proxy artigos científicos) e 0,9937 para pesquisadores, o que demonstra que a distribuição espacial das atividades inovativas no Brasil encontra-se altamente concentrada. O objetivo aqui, portanto, alinha-se ao trabalho de Albuquerque et al. (2001), diferenciando-se deste pela proposta metodológica e pela unidade de análise: as IES federais do nordeste.

Utilizou-se para mensurar a desigualdade, diferentemente de Albuquerque et al. (2001), o índice T de Theil, o qual pode ser deduzido supondo-se uma população com $n$ elementos em que cada elemento apresenta uma fração não negativa de uma variável $Y$ qualquer $\left(Y_{i}, \operatorname{com} i=1, \ldots, n\right)$ : se a média da distribuição de $Y$ é $\mu$ e $X_{i}$ é o valor do $i$-ésimo elemento, $\mathrm{Yi}=\frac{X_{i}}{n \mu}$ e, portanto, $\sum_{i=1}^{n} Y_{i}=1$. Sendo $H(Y)$ definido como a entropia da distribuição, tem-se que $H(Y)=\sum_{i=1}^{n} Y_{i} \log \frac{1}{Y_{i}}$, podendo variar da seguinte forma $0 \leq H(Y) \leq \log (n)$. Se $H(Y)=0$, tem-se a perfeita igualdade e, contrariamente, se $H(Y)=\log (n)$, tem-se a perfeita desigualdade. $\mathrm{O}$ índice $\mathrm{T}$ de Theil é calculado subtraindo a entropia de seu próprio valor Revista Economia \& Tecnologia (RET) 
máximo (Theil, 1967 apud Hoffmann, 2006), como se segue:

$$
\begin{gathered}
T=\log (n)-H(Y) \\
T=\sum_{i=1}^{n} Y_{i} \log (n) . Y_{i} \\
T=\sum_{i=1}^{n} Y_{i} \log \frac{Y_{i}}{1 / n}
\end{gathered}
$$

Dessa forma, tomando-se, por exemplo, a variável grupo de pesquisa, chega-se à seguinte análise: a população é formada por 14 membros, $n$, (todas as universidade federais da região nordeste do Brasil) e cada membro tem uma fração do total de grupos de pesquisa $(Y \geq 0)$. A média de grupos de pesquisa é dado por $\mu$ e $X_{i}$ é o volume de grupos de pesquisa da $i$-ésima instituição. Assim, calcula-se o índice T para o ano 2008. Quanto mais próximo de 0 , mais perfeita a igualdade entre as universidades em questão e quanto mais próximo de 1,1461 , que é exatamente o valor de $\log (14)$, mais desiguais serão as universidades. O mesmo é feito para os demais quesitos, como é apresentado na Tabela 2.

A partir das informações da Tabela 2, pode-se perceber que as universidades federais do nordeste não formam um grupo homogêneo, pois há um considerável grau de desigualdade em todos esses quatro quesitos apresentados.

Tabela 2 - Índice de desigualdade T de Theil dos pesquisadores, grupos de pesquisa, produção bibliográfica e produção técnica das universidades federais do nordeste, 2008

\begin{tabular}{l|c|c|c|c}
\hline & Pesquisadores & $\begin{array}{c}\text { Grupos de } \\
\text { pesquisa }\end{array}$ & $\begin{array}{c}\text { Produção } \\
\text { bibliográfica(*) }\end{array}$ & $\begin{array}{c}\text { Produção } \\
\text { técnica(**) }\end{array}$ \\
\hline T de Theil & 0,11332343 & 0,117474893 & 0,114719992 & 0,181811521 \\
\hline
\end{tabular}

Fonte: Elaboração própria a partir do Plano Tabular do Diretório de Grupos de Pesquisa do CNPq.

Nota: $\left(^{*}\right)$ Artigos publicados em periódicos indexados (nacional e internacional); (**) Produção técnica aqui se refere ao somatório da produção de software e produtos tecnológicos somente com registro de patentes.

Dito isto, a seguir, procura-se caracterizar as universidades federais do nordeste, considerando suas desigualdades e seu papel no sistema de inovação.

\subsection{As grandes áreas do conhecimento nas IES federais do nordeste}

Como grandes áreas de conhecimento, este trabalho optou por utilizar a 
classificação da Coordenação de Aperfeiçoamento de Pessoal de Nível Superior (CAPES). Dessa forma, levaram-se em consideração as seguintes áreas: ciências agrárias; ciências biológicas; ciências da saúde; ciências exatas e da terra; ciências humanas; ciências sociais aplicadas; engenharias; e linguísticas, letras e artes.

O objetivo desta análise é verificar a ocorrência de pesquisas em áreas que possam englobar pesquisas de setores estratégicos para o país. Ao aceitar que tais áreas, como software, fármacos, biotecnologia, biomassa, nanotecnologia, semicondutores e bens de capital, são estratégicas e portadoras de futuro (PITCE, 2003), as quais têm o potencial de mudar radicalmente processos/produtos (Salerno, 2004), tem-se que, ao cruzar essas áreas com as grandes áreas de conhecimento mencionadas acima, se chega ao seguinte quadro-síntese abaixo:

Quadro 1 - Grandes áreas de conhecimento versus áreas da PITCE*

\begin{tabular}{ll}
\hline \multicolumn{1}{c}{ Áreas do conhecimento - CAPES } & Áreas estratégicas em sentido amplo - PITCE \\
\hline Ciências exatas e da terra & Software \\
\hline Engenharias & Semicondutores \\
\hline Ciências da saúde & Bens de capital \\
\hline Ciências agrárias & Fármacos \\
\hline Ciências biológicas & Biomassa \\
\hline Ciências sociais aplicadas & - \\
\hline Ciências humanas & - \\
\hline Linguística, letras e artes & - \\
\hline Multidisciplinar & - \\
\hline
\end{tabular}

Fonte: Chiarini; Vieira, no prelo.

Nota: Embora haja cruzamento direto entre áreas do conhecimento (CAPES) e áreas estratégicas (PITCE), nada impede que pesquisas sobre determinada área estratégica sejam realizadas por pesquisadores de outras áreas do conhecimento. Chiarini e Vieira (no prelo) demonstram que, embora a área estratégica software pertença às ciências exatas e da terra, pesquisas sobre softwares são realizadas por demais áreas, como agrárias, engenharias, ciências da saúde, ciências sociais aplicadas, ciências humanas e até mesmo linguística, letras e artes; ${ }^{*}$ )PITCE refere-se à Política Industrial, Tecnológica e de Comércio Exterior.

Em relação aos grupos de pesquisa divididos por grande área de conhecimento, tem-se que, observando o Gráfico 1, no Brasil, a área de conhecimento com maior número de grupos de pesquisa registrados no Conselho Nacional de Desenvolvimento Científico e Tecnológico (CNPq) é a de ciências humanas (19\%), seguida pela área de saúde (17\%) e engenharias (13\%). Na região nordeste, observa-se que ciências humanas também é a principal área de conhecimento entre os grupos de pesquisa registrados no CNPq (21\%), seguida de saúde 
(16\%) e engenharias (13\%).

Gráfico 1 - Distribuição dos grupos de pesquisa por área de conhecimento, Brasil e Nordeste - 2008
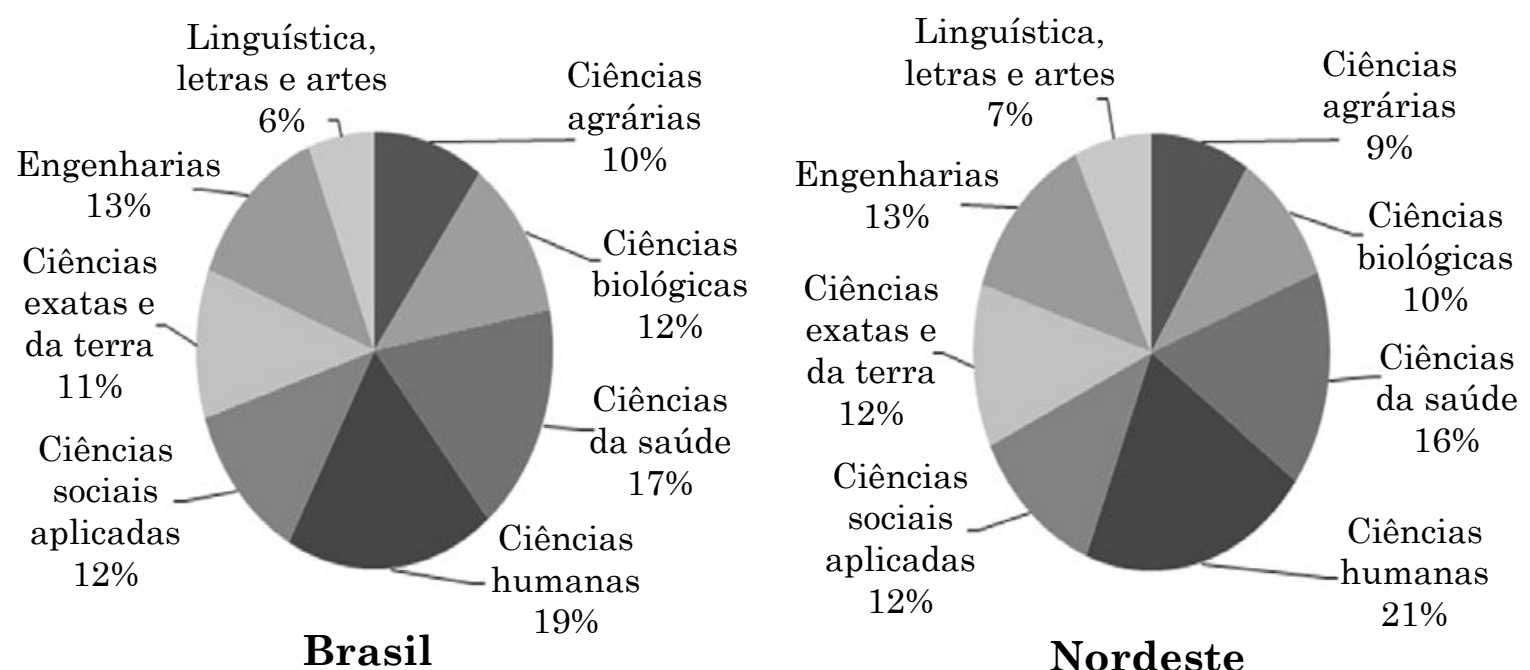

Fonte: Elaboração própria a partir do Plano Tabular do Diretório de Grupos de Pesquisa do CNPq.

Nota: Consideram-se aqui todas as IES públicas e privadas do Brasil.

É válido destacar que, no Brasil, ao se realizar a comprovação que a maior alocação de recursos humanos encontra-se em ciências humanas, sociais Aplicadas e Linguística, Letras e Artes (juntas somam 37\% dos grupos de pesquisa e $35,8 \%$ do número de pesquisadores), nem todo tipo de pesquisa realizada pelas IES direciona-se para setores estratégicos definidos por políticas industriais e de desenvolvimento do país. Na região nordeste, 40\% dos grupos de pesquisa cadastrados no CNPq são de ciências humanas, sociais aplicadas e linguística, letras e artes. Esta distribuição mostra a falta de direcionamento das pesquisas universitárias para áreas científico-tecnológicas.

De certo que não configura entre as intenções deste trabalho sugerir ou defender a exclusão de linhas de pesquisa que não se enquadrem nos setores prioritários das políticas industriais e científico-tecnológicas do país. Entendese que são importantes instrumentos de compreensão da dinâmica regional, histórica, cultural, econômica e social (Chiarini; Vieira, no prelo), no entanto, ressalva-se que a relevância dada às pesquisas das IES voltadas para áreas prioritárias ao desenvolvimento científico-tecnológico vai ao encontro com a temática dos sistemas de inovação e é corroborada pela experiência de países que incentivaram a formação acadêmica em área de ciência e tecnologia, os quais Revista Economia \& Tecnologia (RET) 
estimularam a formação de pessoal qualificado na área de ciência e tecnologia, com ênfase nas áreas de exatas (matemática e engenharia).

\subsection{Recursos humanos para a produção de conhecimentos técnico-científicos}

Para gerar conhecimento científico é preciso mão de obra altamente qualificada, assim a presença dos pesquisadores nas IES se faz mister. A distribuição de pesquisadores entre as universidades federais do nordeste não é homogênea. Em 2008, dos mais de 28 mil pesquisadores ${ }^{8}$ nas universidades nordestinas ${ }^{9}$, aproximadamente $18 \%$ estavam alocados na UFPE, seguida da UFBA, com aproximadamente $17 \%$ de todos os pesquisadores nordestinos das IES federais (Tabela 3). As UFPE, UFBA e UFC concentram 47\% de todos os pesquisadores das IES federais do nordeste.

Tabela 3 - Pesquisadores por IES federal do nordeste, \%, 2008



Fonte: Elaboração própria a partir do Plano Tabular do Diretório de Grupos de Pesquisa do CNPq.

Pode-se ainda analisar a heterogeneidade em termos de capacidade de produção de conhecimento das universidades federais nordestinas segundo a grande área predominante dos pesquisadores (Tabela 4).

\footnotetext{
8 De acordo com informações do $\mathrm{CNPq}$, pesquisador não é sinônimo de professor, já que no Diretório dos Grupos de Pesquisa o termo pesquisador é usado para os membros graduados ou pós-graduados da equipe de pesquisa, diretamente envolvidos com a realização de projetos e com a produção científica, tecnológica e artística do grupo, podendo o pesquisador ser de outra instituição diferente da do líder do grupo. Caso haja alguém matriculado em um curso de graduação ou pós-graduação (especialização, mestrado ou doutorado), ele passa a ser incluído como estudante, desde que seu orientador seja um pesquisador do grupo em questão. Estagiários pós-doutorais, por sua vez, são considerados como pesquisadores do grupo. De acordo com o CNPq, em geral há dupla contagem no número de pesquisadores tendo em vista que o indivíduo que participa de mais de um grupo de pesquisa foi computado mais de uma vez.

9 Considera-se aqui todas as IES públicas e privadas do NE.
}

Revista Economia \& Tecnologia (RET) 
Tabela 4 - Pesquisadores por área e por universidade federal do NE, 2008

\begin{tabular}{l|r|r|r|r|r|rr|r|r}
\hline & Agrárias & Biológicas & Saúde & $\begin{array}{c}\text { Exatas } \\
\text { e da } \\
\text { terra }\end{array}$ & Humanas & $\begin{array}{c}\text { Sociais } \\
\text { aplicadas }\end{array}$ & Eng. & $\begin{array}{c}\text { Linguística, } \\
\text { letras } \\
\text { e artes }\end{array}$ \\
\hline UFAL & 5,7 & 6,2 & 6,2 & 5,1 & 6,0 & 12,4 & 5,8 & 9,9 \\
UFBA & 6,6 & 15,6 & $\mathbf{2 3 , 6}$ & 9,3 & 15,3 & $\mathbf{2 1 , 5}$ & 14,1 & $\mathbf{3 2 , 4}$ \\
UFC & 12,8 & 9,4 & 13,6 & $\mathbf{5 5 , 8}$ & 11,5 & 5,5 & 10,8 & 9,2 \\
UFCG & 8,9 & 2,0 & 1,8 & 2,3 & 4,7 & 3,6 & 14,9 & 3,0 \\
UFERSA & 7,3 & 0,6 & - & 0,7 & - & 1,2 & 1,2 & - \\
UFMA & 3,2 & 4,2 & 6,3 & 2,5 & 6,4 & 4,7 & 2,5 & 1,8 \\
UFPB & 7,7 & 6,9 & 12,7 & 3,8 & 11,4 & 12,6 & 6,0 & 14,6 \\
UFPE & 4,1 & $\mathbf{2 3 , 0}$ & 19,5 & 9,4 & $\mathbf{1 6 , 3}$ & 17,6 & $\mathbf{2 6 , 8}$ & 10,6 \\
UFPI & 8,8 & 7,1 & 3,7 & 2,2 & 5,7 & 4,4 & 0,3 & 3,5 \\
UFRB & 8,7 & 1,0 & 0,5 & 0,4 & 1,7 & 0,5 & - & 1,6 \\
UFRN & 1,5 & 6,2 & 7,6 & 3,6 & 10,8 & 8,5 & 10,4 & 5,3 \\
UFRPE & $\mathbf{1 6 , 5}$ & 11,0 & - & 2,0 & 1,6 & 1,7 & - & 5,0 \\
UFS & 5,0 & 5,2 & 3,5 & 2,6 & 8,2 & 4,9 & 4,8 & 3,1 \\
UNIVASF & 3,1 & 1,5 & 1,0 & 0,3 & 0,4 & 0,9 & 2,4 & - \\
\hline \multicolumn{2}{r}{} & 100 & 100 & 100 & 100 & 100 & 100 & 100 & 100 \\
\hline
\end{tabular}

Fonte: Elaboração própria a partir do Plano Tabular do Diretório de Grupos de Pesquisa do CNPq.

Nota: Em geral há dupla contagem no número de pesquisadores tendo em vista que o indivíduo que participa de mais de um grupo de pesquisa foi computado mais de uma vez.

Com exceção da pesquisa na área de ciências agrárias, em que a UFRPE apresenta tradição e também sinergias devido à própria região onde se localizam seus campi no interior do estado, em Garanhuns, Serra Talhada, Carpina, Ibimirim, Igarassu, Parnamirim e São Lourenço da Mata, as UFBA, UFPE e UFC constituem os principais atores geradores de conhecimento no sistema regional de inovação nordestino. Juntas, as três universidades chegam, em determinadas áreas do conhecimento, a concentrar mais de 50\% dos pesquisadores, como é o caso das engenharias, com $51,7 \%$ dos pesquisadores, saúde, com 56,7\% e exatas e da terra, com 74,5\%, áreas que apresentam papel estratégico para o posicionamento em novos paradigmas técnico-econômicos.

A quantidade de programas de pós-graduação de qualidade afeta igualmente a produção de conhecimento. A geração de novos conhecimentos relevantes tem seu principal suporte no sistema público de pesquisa, fruto da pós-graduação (Marcovith, 1999), por isso faz-se necessário saber qual a quantidade e qualidade de cursos de mestrado acadêmico, mestrado profissional e doutorado oferecidos nas IES federais do nordeste. Em 2008, foram ofertados 348 programas, sendo que aproximadamente $47 \%$ desses estavam concentrados em três universidades: UFPE, UFBA e UFC (Tabela 5). No entanto, no nordeste existia apenas um curso que obteve a qualidade máxima, o Programa de Mestrado/ 
Doutorado em Astronomia/Física da UFPE.

Tabela 5 - Programas de pós-graduação, IES federais do nordeste, \%, 2008

\begin{tabular}{lrr}
\hline \multicolumn{1}{c|}{ IES } & $\mathbf{\%}$ \\
\hline UFPE & & $\mathbf{1 6 , 7}$ \\
UFBA & & $\mathbf{1 5 , 2}$ \\
UFC & & $\mathbf{1 5 , 2}$ \\
UFRN & 11,2 \\
UFPB & 10,6 \\
UFRPE & 5,5 \\
UFAL & 5,2 \\
UFCG & 4,9 \\
UFS & 4,9 \\
UFPI & 4,0 \\
UFMA & & 3,7 \\
UFERSA & & 1,1 \\
UFRB & & 1,1 \\
UNIVASF & & 0,6 \\
\hline
\end{tabular}

Fonte: Elaboração própria a partir dos dados estatísticos do GeoCapes.

Nota: Inclui programas de mestrado acadêmico, mestrado profissional e doutorado.

Lançando-se mão mais uma vez da base de dados do Diretório de Grupos de Pesquisa do $\mathrm{CNPq}$, é possível verificar o número de grupos de pesquisa nas IES federais do nordeste, também em 2008 (Tabela 6). Pode-se notar a partir dos dados apresentados na Tabela 6 que as UFPE, UFBA e UFC centralizam aproximadamente $47 \%$ de todos os grupos de pesquisa das IES federais do nordeste. As Tabelas 5 e 6 corroboram o fato de existir concentração de recursos de pesquisa nas IES federais do nordeste.

Tabela 6 - Grupos de pesquisa por IES federal do nordeste, \%, 2008

\begin{tabular}{l|r}
\hline \multicolumn{1}{c|}{ IES } & $\mathbf{1 0}$ \\
\hline UFPE & $\mathbf{1 9 , 4}$ \\
UFBA & $\mathbf{1 7 , 0}$ \\
UFC & $\mathbf{1 0 , 7}$ \\
UFPB & 10,2 \\
UFAL & 8,8 \\
UFRN & 6,6 \\
UFPI & 5,3 \\
UFCG & 5,3 \\
UFS & 5,1 \\
UFMA & 4,6 \\
UFRPE & 3,2 \\
UNIVASF & 1,5 \\
UFRB & 1,3 \\
UFERSA & 1,1 \\
\hline Fonte: Elaboração própria a partir do Plano Tabular do Diretório de Grupos \\
de Pesquisa do CNPq.
\end{tabular}




\subsection{Esforços na produção de conhecimentos técnico-científicos}

O quesito output, ou seja, dados referentes à produção científica e tecnológica (C\&T), que pode ser desagregada em produção bibliográfica (artigos completos publicados em periódicos nacionais e internacionais indexados) e produção técnica propriamente dita (produção de software, produtos tecnológicos e processos ou técnicas, com ou sem registro), é importante para analisar o desempenho das IES em questão. Levando-se em consideração a produção bibliográfica, isto é, o somatório de artigos completos publicados em periódicos especializados nacionais e internacionais de pesquisadores e estudantes, mais uma vez, verifica-se concentração em três universidades: UFPE, UFBA e UFC, as quais aglomeram cerca de $48 \%$ de toda produção bibliográfica das IES federais dessa região (Tabela 7). No entanto, vale ressaltar que as IES federais do nordeste contribuem apenas com aproximadamente $14 \%$ dos artigos publicados nacionalmente em periódicos indexados e com cerca de $12 \%$ dos artigos internacionalmente publicados.

Tabela 7 - Produção bibliográfica por IES federal do nordeste, \%, 2008

\begin{tabular}{|c|c|c|}
\hline IES & IES & $\%$ \\
\hline$\overline{\mathrm{UFPE}}$ & 17,8 UFCG & 4,6 \\
\hline UFBA & 16,1 UFS & 4,0 \\
\hline UFC & 14,0 UFMA & 3,9 \\
\hline UFPB & 11,1 UFRPE & 3,3 \\
\hline UFAL & 8,0 UNIVASF & 2,3 \\
\hline UFRN & 6,4 UFRB & 1,7 \\
\hline UFPI & 5,9 UFERSA & 1,0 \\
\hline
\end{tabular}

Fonte: Elaboração própria a partir do Plano Tabular do Diretório de Grupos de Pesquisa do CNPq.

Nota: Produção bibliográfica refere-se ao somatório de artigos completos publicados em periódicos especializados nacionais e internacionais de pesquisadores e estudantes. Não há dupla contagem nos quantitativos da produção.

Analisando o output produção técnica, levando-se em conta a produção de software (com/sem registro de patentes), produtos tecnológicos (com/sem registro de patentes) e processos/técnicas (com/sem registro em catálogo) tem-se que as IES federais do nordeste apropriam-se pouco do processo de produção técnico brasileiro. Para se ter uma ideia, em 2008, a produção de software com registro de patentes pelas IES federais do nordeste representou cerca de 5,6\% da produção nacional.

O mesmo pode ser dito dos produtos tecnológicos com registro de paten- 
tes: as IES federais do nordeste concentraram apenas $10 \%$. No entanto, entre as IES federais do nordeste, as UFPE, UFC e a UFBA se destacam na produção de software com registro de patentes, com 57,3\% do total das IES nordestinas (Tabela 8). Em relação aos produtos tecnológicos com registro de patentes, tem-se que as UFPE, UFC e UFBA concentram $56,7 \%$ da produção técnica dessa categoria de todas as IES federais do nordeste (Tabela 9), mas, mais uma vez, o bom desempenho dessas três instituições não garante um bom posicionamento do nordeste em relação ao restante do país; dessa forma, as IES federais do nordeste concentram apenas $9 \%$ de produtos tecnológicos com registro de patentes.

Tabela 8 - Produção técnica (software) por IES federal do nordeste, \%, 2008

\begin{tabular}{l|r|r|r}
\hline & $\begin{array}{c}\text { Participação } \\
\text { total (\%) }\end{array}$ & $\begin{array}{c}\text { Sem patente } \\
\text { (\%) }\end{array}$ & $\begin{array}{c}\text { Com patente } \\
\text { (\%) }\end{array}$ \\
\hline UFPE & 21,6 & 12,5 & $\mathbf{2 2 , 1}$ \\
UFC & 18,5 & 3,1 & $\mathbf{1 9 , 3}$ \\
UFBA & 16,5 & 28,1 & $\mathbf{1 5 , 9}$ \\
UFAL & 10,9 & 3,1 & 11,3 \\
UFCG & 7,5 & 12,5 & 7,2 \\
UFPB & 5,9 & 21,9 & 5,1 \\
UFS & 5,4 & 0,0 & 5,7 \\
UFRN & 5,1 & 9,4 & 4,9 \\
UFRPE & 2,6 & 6,3 & 2,5 \\
UFMA & 2,6 & 0,0 & 2,8 \\
UFERSA & 1,9 & 3,1 & 1,8 \\
UFPI & 0,8 & 0,0 & 0,8 \\
UFRB & 0,6 & 0,0 & 0,7 \\
UNIVASF & 0,0 & 0,0 & 0,0 \\
\hline Fin
\end{tabular}

Fonte: Elaboração própria a partir do Plano Tabular do Diretório de Grupos de Pesquisa do CNPq.

Tabela 9 - Produção técnica (produtos tecnológicos) por IES federal do nordeste, \%, 2008

\begin{tabular}{l|rrr}
\hline & $\begin{array}{c}\text { Participação } \\
\text { total (\%) }\end{array}$ & \multicolumn{1}{c|}{$\begin{array}{c}\text { Sem } \\
\text { patente (\%) }\end{array}$} & $\begin{array}{c}\text { Com } \\
\text { patente (\%) }\end{array}$ \\
\hline UFPE & 23,0 & 24,3 & $\mathbf{2 3 , 8}$ \\
UFC & 16,0 & 16,4 & $\mathbf{1 6 , 2}$ \\
UFBA & 14,9 & 17,9 & $\mathbf{1 6 , 7}$ \\
UFRN & 11,0 & 8,6 & 9,6 \\
UFPB & 10,3 & 7,4 & 8,6 \\
UFAL & 5,7 & 3,7 & 4,5 \\
UFRB & 4,3 & 5,4 & 4,9 \\
UFPI & 3,9 & 3,2 & 3,5 \\
UFS & 3,9 & 4,4 & 4,2 \\
UFCG & 2,5 & 4,2 & 3,5 \\
UFRPE & 2,5 & 3,4 & 3,0 \\
UNIVASF & 1,4 & 0,2 & 0,7 \\
UFERSA & 0,4 & 0,0 & 0,1 \\
UFMA & 0,4 & 1,0 & 0,7 \\
\hline
\end{tabular}

Fonte: Elaboração própria a partir do Plano Tabular do Diretório de Grupos de Pesquisa do CNPq. 


\subsection{Relação entre os grupos de pesquisa e as empresas}

Dos 292 grupos declarados pelas IES federais do nordeste que possuem algum tipo de relacionamento com empresas, a maioria concentra-se nas UFPE, UFBA e UFC, cada uma apropriando-se com $26 \%, 19,5 \%$ e $10,3 \%$ respectivamente do total das IES federais do nordeste (Gráfico 2).

Gráfico 2 - Proporção dos grupos de pesquisa relacionados com empresas. por IES federal do nordeste. 2008

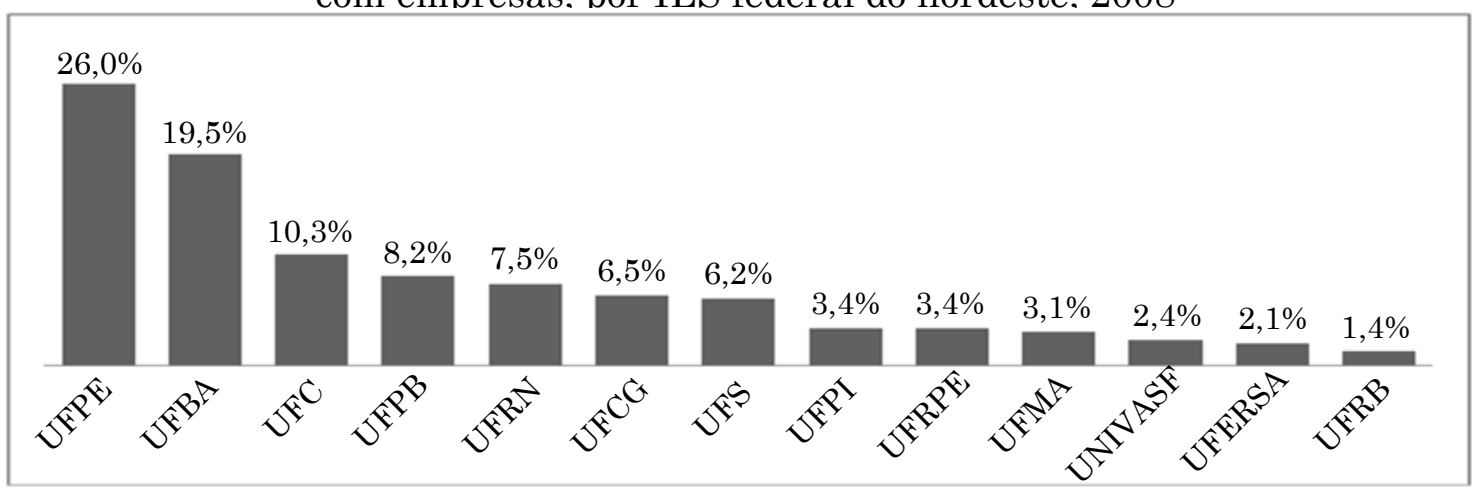

Fonte: Elaboração própria a partir do Plano Tabular do Diretório de Grupos de Pesquisa do CNPq.

Entre os possíveis propósitos de relacionamentos ${ }^{10}$ grupos-empresas, a pesquisa científica com considerações de uso imediato dos resultados é a que mais se destaca, com $27,5 \%$. Outro resultado que se destaca é o relacionamento grupo-empresa para fins de transferência de tecnologia desenvolvida pelo grupo para a empresa, 15\%. Em contrapartida, as empresas auxiliam ${ }^{11}$ em sua maioria as IES federais do nordeste, com transferência de recursos financeiros da empresa para o grupo (26\%), transferência de insumos materiais para as 10 De acordo com o Diretório dos Grupos de Pesquisa do CNPq, os relacionamentos grupos/empresas podem ser classificados em 14 tipos: pesquisa científica sem considerações de uso imediato dos resultados; pesquisa científica com considerações de uso imediato dos resultados; atividades de engenharia não rotineira inclusive o desenvolvimento de protótipo cabeça de série ou planta-piloto para o parceiro; atividades de engenharia não rotineira inclusive o desenvolvimento/fabricação de equipamentos para o grupo; desenvolvimento de software não rotineiro para o grupo pelo parceiro; desenvolvimento de software para o parceiro pelo grupo; transferência de tecnologia desenvolvida pelo grupo para o parceiro; transferência de tecnologia desenvolvida pelo parceiro para o grupo; atividades de consultoria técnica não contempladas nos demais tipos; fornecimento, pelo parceiro, de insumos materiais para as atividades de pesquisa do grupo sem vinculação a um projeto específico de interesse mútuo; fornecimento, pelo grupo, de insumos materiais para as atividades do parceiro sem vinculação a um projeto específico de interesse mútuo; treinamento de pessoal do parceiro pelo grupo incluindo cursos e treinamento "em serviço"; treinamento de pessoal do grupo pelo parceiro incluindo cursos e treinamento "em serviço"; outros tipos predominantes de relacionamento que não se enquadrem em nenhum dos anteriores.

11 De acordo com o Diretório dos Grupos de Pesquisa do CNPq, as remunerações empresas/grupos podem ser classificadas em 10 categorias: transferência de recursos financeiros do parceiro para o grupo; transferência de recursos financeiros do grupo para o parceiro; fornecimento de bolsas para o grupo pelo parceiro; parceria sem a transferência de recursos de qualquer espécie envolvendo exclusivamente relacionamento de risco; transferência de insumos materiais para as atividades de pesquisa do grupo; transferência de insumos materiais para as atividades do parceiro; transferência física temporária de recursos humanos do parceiro para as atividades de pesquisa do grupo; transferência física temporária de recursos humanos do grupo para as atividades do parceiro; parceria com transferência de recursos de qualquer espécie nos dois sentidos e outras formas de remuneração que não se enquadrem em nenhuma das anteriores.

Revista Economia \& Tecnologia (RET)

Vol. 8(1), p. 137-160, Jan/Mar 2012 
atividades de pesquisa do grupo (20\%) e fornecimento de bolsas para o grupo pelo parceiro (13\%). No entanto, um percentual alto de relacionamento resulta em parcerias sem a transferência de recursos de qualquer espécie envolvendo exclusivamente relacionamento de risco (14\%).

\section{Considerações finais}

Este artigo parte da ideia de que o conhecimento tem se tornado fator de competitividade e elemento sine qua non tanto para a capacidade inovativa quanto para a capacidade de desenvolvimento, seja regional ou nacional. Características sociais, políticas, culturais e institucionais têm papel ativo em moldar o ambiente inovativo e muitos são os atores que devem ser levados em consideração, além do emaranhado de relações possíveis entre eles, para uma política eficaz pró-desenvolvimento.

Um desses atores é a universidade, entendida nesse artigo como instituição cujo papel social vai além de simplesmente formar uma população mais "iluminada", nas palavras de Nowotny et al. (2001), mas produzir conhecimento científico e tecnológico. O enfoque dado, portanto, foi sobre o desempenho das universidades em gerar conhecimento científico e tecnológico, já que outros agentes podem se apropriar dele e conseguir inovar. Esse processo, no entanto, pode não ocorrer de forma direta, ou seja, podem existir dificuldades tangentes à formação de links universidade-empresa relacionadas a sua apropriabilidade.

A partir desse referencial teórico, foi apresentado um retrato da alocação de recursos públicos nas universidades federais nordestinas a fim de ter maior conhecimento de um dos players do sistema nordestino de inovação. Buscou-se, portanto, mapear algumas características das universidades federais nordestinas a fim de contribuir para o amplo debate a respeito da forma eficiente de alocação de recursos públicos voltados para o ensino de nível superior e para pesquisa acadêmica.

Através de tal caracterização, e do cálculo do índice T de Theil, mostrouse que há diferenças entre as universidades federais do nordeste. Da mesma forma observada para o Brasil, a grande área de conhecimento com maior número de grupos de pesquisa registrados no $\mathrm{CNPq}$, na região nordeste, é a área de ciências humanas. Na região nordeste, os grupos de pesquisa em ciências 
humanas, sociais aplicadas e linguística, letras e artes consistem em cerca de $40 \%$ do total de grupos de pesquisa cadastrados no CNPq.

É importante dizer que não é o objetivo deste artigo defender a extinção das pesquisas em áreas não tecnológicas, mesmo porque há o entendimento de que tais pesquisas são importantes para aprofundar o conhecimento histórico, cultural, social e econômico sobre o país. Porém, tal distribuição dos grupos de pesquisa é de certo modo preocupante e deve ser vista com cautela. Um maior direcionamento das pesquisas para áreas estratégicas é fundamental para fortalecer as bases do desenvolvimento, não apenas regional, mas também nacional. Esta iniciativa mostrou-se bastante positiva em países como a Índia (no setor de software) e Cuba (em ciências da saúde).

No que se refere à produção científica (produção bibliográfica e produção técnica), a tríade (formada pelas UFPE, UFBA e UFC) se apresentou como principal agente regional, confirmando que há desigualdade entre as IES federais do nordeste, o que foi corroborado na análise dos dados apresentada nesse trabalho. A tríade concentra aproximadamente $47 \%$ dos pesquisadores das IES federais nordestinas, $47 \%$ dos programas de pós-graduação, $47 \%$ de todos os grupos de pesquisa, $48 \%$ da produção bibliográfica, $53 \%$ da produção de software com registro de patentes e $56 \%$ da produção técnica com registro de patentes de todas IES federais do nordeste.

Considerando-se a produção de software (com/sem registro de patentes), produtos tecnológicos (com/sem registro de patentes) e processos/técnicas (com/sem registro em catálogo), tem-se que as IES federais do nordeste, em geral, apropriam-se pouco do processo brasileiro de produção técnica. Tal cenário merece ser observado cuidadosamente, a fim de que sejam tomadas medidas específicas que visem fortalecimento das universidades federais existentes na região, já que estas podem e devem ir além da formação de mão de obra qualificada. As universidades federais precisam ser incluídas em um plano maior de desenvolvimento, com incentivo à formação de pessoas e à pesquisa em áreas estratégicas como software, biotecnologia, fármacos, entre outras áreas portadoras de futuro e estratégicas para o desenvolvimento da região. As políticas públicas voltadas às IES devem, portanto, ser pensadas em sentido amplo; dessa forma, deve haver um alinhamento com meios que melhorem as relações universidade-empresa-Estado, propiciando campo para o amadurecimento do 
sistema de inovação.

No caso da região nordeste, o direcionamento para áreas estratégicas é importante para sustentar a demanda por tecnologia e recursos humanos qualificados das empresas que têm se instalado na região. Ademais, a formação de profissionais em áreas fundamentais no atendimento à sociedade, como é o caso de profissionais de saúde, principalmente médicos e enfermeiros, é algo indispensável para que a região atinja um crescimento sustentável, compatível com o desenvolvimento.

Um próximo passo, dando continuidade à pesquisa aqui realizada, deve tentar buscar entender o perfil das empresas localizadas no nordeste, podendo, então, confrontar com o perfil da produção de conhecimentos técnicos-científicos nas IES ali localizadas. A identificação de possíveis gaps entre atuação das empresas versus áreas das pesquisas realizadas pelas IES poderá possibilitar a implementação de políticas que incentivem pesquisas específicas para que o conhecimento de base seja melhor aproveitado pelo setor produtivo, levando, possivelmente, a um maior interesse por parte das empresas em investir na pesquisa acadêmica, fazendo com que a retroalimentação seja mais intensa, gerando uma situação win-win.

\section{Referências}

Abdalla, M. M.; Calvosa, M. V. D.; Batista, L. G. (2009). "Hélice tríplice no Brasil: ensaio teórico acerca dos benefícios da entrada da universidade nas parcerias estatais". Cadernos de Administração 3.

Albuquerque, E. M. (1996). "Sistema nacional de inovação no Brasil: uma análise introdutória a partir de dados disponíveis sobre a ciência e a tecnologia”. Revista de Economia Política 16 (3): 56-72.

Albuquerque, E. M. (1998). "Produção científica e sistema nacional de inovação". Ensaios FEE 19(1): 156-180.

Albuquerque, E. M.; Simões, R.; Baessa, A.; Campolina, B.; Silva, L. (2001). “A distribuição espacial da produção científica e tecnológica brasileira: uma descrição de estatísticas de produção local de patentes e artigos científicos". In XXIX Encontro Nacional de Economia.

Albuquerque, E. M. e; Silva, L., A. Póvoa, L. M. C. (2005). "Diferenciação intersetorial na interação entre empresas e universidades no Brasil”. São Paulo em Perspectiva 19(1): 95-104.

Albuquerque, E., M.; Silva, L. A.; Rapini, M. S.; Souza, S. G. A.(2005). "Interactions 
between firms and universities in an immature system of innovations: a survey of industrial R\&D-performers firms in Minas Gerais". Texto para discussão UFMG/Cedeplar, 280.

Almeida, M. (2004). A evolução do movimento de incubadoras no Brasil. Rio de Janeiro: tese de Doutorado, COPPE-UFRJ.

Almeida, M. (2005). "The evolution of the incubator movement in Brazil". In $I V$ Conferencia de Investigación en Entrepreneurship en Latinoamérica (CIELA). URL [on-line]: http://www,icesi,edu,co/ciela/anteriores/Papers/ppem/2,pdf. Acesso em: 10 de setembro de 2010 .

Baldini, J. P.; Borgonhoni, P. (2007). "As relações universidade-empresa no Brasil: surgimento e tipologias”. Caderno de Administração 15(2): 29-38.

Calvosa, M. V. D.; Gouveia, L. B.; Moutinho, M. A. (2009). Parcerias público-privadas e hélice tríplice no Brasil e na América Latina: o benefício da entrada de atores de desenvolvimento em um processo falente do Estado. In 6th International Meeting of the Iberoamerican Academy of Management.

Chiarini, T.; Vieira, K. P.; Zorzin, P. L. (2010). "Produção de pesquisa científica e de conhecimento: um retrato da atual distribuição de recursos entre as universidades federais mineiras". In XIV Seminário sobre a Economia Mineira.

Chiarini, T.; Vieira, K. P.; Zorzin, P. L. (2012). "Universidades federais mineiras: análise da produção de pesquisa científica e conhecimento no contexto do sistema mineiro de inovação". Revista Nova Economia, no prelo.

Chiarini, T.; Vieira, K. P. (2011). "Alinhamento das atividades de pesquisa científica e tecnológica realizadas pelas IES federais de MG e as diretrizes da Política Industrial e de Comércio Exterior". Revista Brasileira de Inovação 10: 301-342.

Cohen. W. et al. (2002). "Link and impacts: the influence of public research on industrial R\&D”. Management Science 48(1): 1-23.

Cruz, C. H. B. (2004). "A universidade, a empresa e a pesquisa". In Seminário Brasil em Desenvolvimento da Universidade Federal do Rio de Janeiro. URL [on-line]: http://www.ifi.unicamp.br/ brito/artigos/univ-empr-pesq-rev102003b.pdf. Acesso em: 28 de janeiro de 2010 .

Dagnino, R.; Gomes, E. J. (2003). "A relação universidade-empresa: comentários sobre um caso atípico”. Gestão e Produção 10(3): 283-292.

Dagnino, R. (2003). "A relação universidade-empresa no Brasil e o 'argumento da Hélice Tripla”. Revista Brasileira de Inovação 2(2); 267-307.

Esteves, L. A. ; Meirelles, J. G. P. (2009). "Innovation and cooperation between firms and universities: evidence from Brazil". In Third Conference on Micro Evidence on Innovation and Development - MEIDE.

Etzkowitz, H.; Webster, A.; Gebhart, C.; Terra, B. (2000). "The future of the university and the university of the future: the evolution of the ivory tower to entrepreneurial paradigm”. Research Policy 29(2).

Etzkowitz, H. (2003). "Innovation in innovation: the triple helix of universityindustry-government relations". Social Science Information 42(3).

Gomes, E. J. (2001). A relação universidade-empresa no Brasil: testando hipóteses a partir do caso da Unicamp. Campinas: tese de doutorado, Programa de Pós- 
graduação em Política Científica e Tecnológica do Instituto de Geociências da Universidade Estadual de Campinas (Unicamp).

Gibbons, M. et al. (1994). The new production of knowledge: the dynamics of science and research in contemporary societies. London: SAGE.

Hagedoorn J.; Link, A. N.; Vonortas, N. S. (2000). "Research partnerships". Research Policy 29: 567-586.

Hoffmann, R. (2006). Estatística para economistas. $4^{\text {a }}$ ed. São Paulo: Cengage.

Kupfer, D. (1996). "Uma abordagem neo-schumpeteriana da competitividade industrial”. Ensaios FEE 1(17): 355-372.

Leydesdorff, L.; Etzkowitz, H. (1998). “The triple helix as a model for innovation studies". Science \& Public Policy 25(3).

Leydesdorff, L. (200). "The triple helix: an evolutionary model of innovations". Research Policy 29.

Leydesdorff, L.; Meyer, M. (2006). "The triple helix indicators of knowledge-based innovation systems: introduction to special issue". Research Policy 35.

Marcovitch, J. (1999). "A cooperação da universidade moderna com o setor empresarial”. Revista de Administração 34(4).

Marques, R. A.; Freitas, I. M. B.; Silva, E. M. P. (2007). "Colaboração com universidade e as atividades para inovação de empresas brasileiras". Engevista 9(2): $112-128$.

Mello, J. M. C. (2004). "A abordagem hélice tríplice e o desenvolvimento regional”. II Seminário Internacional Empreendedorismo, Pequenas e Médias Empresas e Desenvolvimento Local.

Mello, J. M. C.; Maculan, A. M.; Renault, T. (2009). "Brazilian universities and their contribution to innovation and development". In Bo Goransson; Claes Brundenius. (Org.). Developing universities: the changing role of academic institutions in development. Londres: Routledge/IDRC.

Mowery, D.; Sampat, B. (2005). "Universities in national innovation systems". In Fagerberg, J.; Mowery, D.; Nelson. R. The Oxford handbook of innovation. Oxford: Oxford University Press.

Nowotny, H. et al. (2001). Re-thinking science: knowledge and the public in an age of uncertainty. Cambridge: Polity Press.

Política Industrial, Tecnológica e de Comércio Exterior. (2003). Diretrizes de política industrial, tecnológica e de comércio exterior. URL [on-line]: http://www.abdi.com. $\mathrm{br} / \mathrm{q}=$ =system/files/diretriz_politica_industrial.pdf . Acesso em: 24 de junho de 2010.

Plonsky, G. A. (1995). "Cooperação empresa-universidade: antigos dilemas, novos desafios”. Revista USP: Dossiê Universidade -Empresa 25: 32-41.

Povoa, L. C.; Rapini, M. S. (2009). “Technology transfer from universities and public research institutes to firms in Brazil: what is transferred and how the transfer is made". In XXXVII Encontro Nacional de Economia.

Rapini, M. S.; Campos, B. C. (2004). "As universidades mineiras e suas interações com a indústria: uma análise a partir de dados do diretório". In XI Seminário 
sobre a Economia Mineira.

Rapini, M. S.; Albuquerque, E., M.; Silva, L.; Souza, S. G. A.; Righi, H. M.; Cruz, W. M. S. (2006). "Spots of interactions: an investigation on the relationship between firms and universities in Minas Gerais". Texto para discussão UFMG / Cedeplar 286.

Rapini, M. S. (2007). "Interação universidade-empresa no Brasil: evidências do Diretório dos Grupos de Pesquisa do CNPq". Estudos Econômicos 37: 211-233.

Rapini, M. S.; Righi, H. M. (2007). "Interação universidade-empresa no Brasil em 2002 e 2004: uma aproximação a partir dos grupos de pesquisa do CNPq". Revista Economia 8(2): 248-268.

Rapini, M. S.; Suzigan, W.; Fernandes, R.; Domingues, E.; Carvalho, S. S. M.; Chaves, C., V. (2009). "A contribuição das universidades e institutos de pesquisa para o sistema de inovação brasileira”. In XXXVII Encontro Nacional de Economia.

Renault, Thiago; Mello, J. M. C. ; Carvalho, R. (2008). "Universities, organizational structure of the research activity and the spin-off formation: lessons from the brazilian case". In XXV IASP World Conference.

Righi, H. M.; Rapini, M. S. (2006). "A evolução da interação entre universidade e empresas em Minas Gerais: uma análise a partir dos censos 2002 e 2004 do Diretório dos Grupos de Pesquisa do CNPq". In XII Seminário sobre a Economia Mineira.

Sabato, J. A.; Botana, N. (1968). "La ciencia y la tecnología en el desarrollo futuro de América Latina”. Revista de la Integración 3: 15-36.

Salerno, M. S. (2004). "A política industrial, tecnológica e de comércio exterior do governo federal”. Parcerias Estratégicas 19.

Silva, L.; Rapini, M. S.; Fernandes, R.; Verona, A. P. (2000). "Estatísticas de patentes e atividades tecnológicas em Minas Gerais". In IX Seminário sobre a Economia Mineira.

Suigan, W.; Albuquerque, E. M. (2008). "A interação entre universidades e empresas em perspectiva histórica no Brasil”. Texto para discussão UFMG/Cedeplar 329.

Suzigan, W.; Albuquerque, E. M. (2009). "The underestimated role of universities for development: notes on historical roots of Brazilian system of innovation". In XVth World Economic History Congress.

Theis, I. M. et al. (2006). "A contribuição da universidade para sistemas regionais de inovação: o caso da Furb”. Revista Economia \& Gestão da PUC Minas 06(12).

Vasconcelos, M. C. R. L.; Ferreira, M. A. T. (2000). "A contribuição da cooperação universidade/empresa para o conhecimento tecnológico da indústria”. Perspectivas em Ciencia da Informação 5(2): 167-182.

Vieira, K. P.; Albuquerque, E. M. (2007). "O financiamento às atividades inovativas na região nordeste: uma análise descritiva a partir dos dados da Pintec". Revista Econômica do Nordeste 38(3).

Viotti, E. B. (2010). "Doutores 2010: estudos da demografia da base técno-científica brasileira". In Doutores 2010: estudos da demografia da base técnico-científica brasileira. Brasília: Centro de Gestão e Estudos Estratégicos. 
Tulio Chiarini, Karina Pereira Vieira

Zawislak, P. A; Dalmarco, G. (2009). "University-industry interaction: a knowledge flow typology". In 18th International Conference on Management of Technology. 